Research Article

\title{
Flux Density through Guides with Microstructured Twisted Clad DB Medium
}

\author{
M. A. Baqir and P. K. Choudhury \\ Institute of Microengineering and Nanoelectronics, Universiti Kebangsaan Malaysia, UKM, Bangi 43600, Selangor, Malaysia \\ Correspondence should be addressed to P. K. Choudhury; pankaj@ukm.my
}

Received 21 October 2013; Accepted 2 December 2013; Published 2 January 2014

Academic Editor: Razali Ismail

Copyright (C) 2014 M. A. Baqir and P. K. Choudhury. This is an open access article distributed under the Creative Commons Attribution License, which permits unrestricted use, distribution, and reproduction in any medium, provided the original work is properly cited.

\begin{abstract}
The paper deals with the study of flux density through a newly proposed twisted clad guide containing DB medium. The inner core and the outer clad sections are usual dielectrics, and the introduced twisted windings at the core-clad interface are treated under DB boundary conditions. The pitch angle of twist is supposed to greatly contribute towards the control over the dispersion characteristics of the guide. The eigenvalue equation for the guiding structure is deduced, and the analytical investigations are made to explore the propagation patterns of flux densities corresponding to the sustained low-order hybrid modes under the situation of varying pitch angles. The emphasis has been put on the effects due to the DB twisted pitch on the propagation of energy flux density through the guide.
\end{abstract}

\section{Introduction}

Metamaterials are artificially designed mediums that owe unusual phenomena, such as reversal of Snell's law and Doppler's effect, negative reflection/refraction, and many others. These exotic features are basically due to the structures of materials rather than their compositions, and are used in many interesting applications, for example, cloaking, perfect lensing, and power confinement [1-4]. References [5, 6] describe negative index chiral metamaterial based on eightcrank molecule designed structure; homogeneous as well as isotropic behavior of chiral medium, based on the periodic inclusion of cranks, have been demonstrated.

During the last couple of decades, complex structured guides have attracted the R\&D community, primarily because of their varieties of potential applications that include optical sensing, integrated optics, and microwaves devices. The electromagnetic behavior of guides can be tailored by altering structural geometry, medium composition, and the nature of excited electromagnetic field. References [7-11] describe several forms of waveguide structures and their electromagnetic response. Reference [8] demonstrates wave propagation through chiral nihility metamaterial, a special class of chiral medium, in which the real part of permittivity and permeability simultaneously becomes zero, while its chirality remains nonzero. In the context of complex optical micro- and/or nanostructures, helical clad optical guides offer control over the dispersion behavior through suitable adjustments of the helix pitch angle [12-16]. Such helical forms or the twists can be written on fiber structures by the use of current advancements in nanotechnology.

The usefulness of DB boundary conditions in the construction of spherical and cylindrical cloaks has been reported in the literature [3]. Within the context, the DB boundary conditions were introduced by Lindell et al. [1719], in which the normal components of both electric and magnetic fields vanish at the interface [20-22]. DB medium can be easily realized in the case of anisotropic medium if the normal component of permittivity and permeability simultaneously becomes zero; that is,

$$
\widehat{n} \cdot \vec{B}=0, \quad \widehat{n} \cdot \vec{D}=0
$$

In the case of isotropic medium, the boundary conditions can be written as

$$
\widehat{n} \cdot \vec{H}=0, \quad \widehat{n} \cdot \vec{E}=0
$$


In the present paper, we investigate the propagation through twisted clad guiding medium, wherein helical windings are introduced as DB medium at the dielectric coreclad interface. To the best of our knowledge, such guiding structures are not yet discussed in the literature, and the present work would be the first investigation of the fundamental electromagnetic behavior of microstructured guides with DB medium twisted clad. Rigorous analyses are made of the associated boundary-value problem implementing Maxwell's equations, and the dispersion relations of the guide are deduced followed by the estimation of supported flux density patterns.

\section{Theoretical Approach}

We consider a cylindrical twisted clad guiding medium, as shown in Figure 1, the core/clad sections of which are linear, isotropic, homogeneous, and lossless dielectrics. A DB based microstructured sheath helix is introduced at the core-clad interface, treated under the suitable boundary conditions. Although the sheath is tightly wound, each of the turns is insulated from the neighboring ones. In Figure 1, $\Psi$ represents the angle that the conducting helical windings make with respect to the normal drawn on to the fiber surface in the longitudinal direction.

We assume $n_{\text {core }}$ and $n_{\text {clad }}$ as the refractive indices (RIs) of core and clad regions of the guide, respectively, and $a$ as the core radius, the clad section being infinitely extended. The time $t$-harmonic and axis $z$-harmonic wave propagates down the guiding structure, considering the guide to be elongated along the $z$-direction. Such an assumption leads to the transverse electric/magnetic field components to be of the forms as

$$
\begin{aligned}
& E_{r 1}=-\frac{j}{u^{2}}\left[\beta u A J_{v}^{\prime}(u r)+\frac{j \omega \mu v}{r} B J_{v}(u r)\right] e^{j(\omega t-\beta z+v \varphi)}, \\
& H_{r 1}=\frac{j}{u^{2}}\left[\frac{j \omega \mu v}{r} A J_{v}(u r)-\beta u B J_{v}^{\prime}(u r)\right] e^{j(\omega t-\beta z+v \varphi)}, \\
& E_{\varphi 1}=-\frac{j}{u^{2}}\left[\frac{j \beta v}{r} A J_{v}(u r)-\omega \mu u B J_{v}^{\prime}(u r)\right] e^{j(\omega t-\beta z+v \varphi)}, \\
& H_{\varphi 1}=-\frac{j}{u^{2}}\left[\omega \mu u A J_{v}^{\prime}(u r)+\frac{j \beta v}{r} B J_{v}(u r)\right] e^{j(\omega t-\beta z+v \varphi)},
\end{aligned}
$$

wherein $u=\sqrt{\left(n_{\text {core }} k_{0}\right)^{2}-\beta^{2}}$. Similarly, the transverse components of fields in the clad section can be written as

$$
\begin{aligned}
& E_{r 2}=-\frac{j}{w^{2}}\left[\beta w C K_{v}^{\prime}(w r)+\frac{j \omega \mu v}{w} D K_{v}(w r)\right] e^{j(\omega t-\beta z+v \varphi)}, \\
& H_{r 2}=\frac{j}{w^{2}}\left[\frac{j \omega \mu v}{r} C K_{v}(w r)-\beta w D K_{v}^{\prime}(w r)\right] e^{j(\omega t-\beta z+v \varphi)},
\end{aligned}
$$

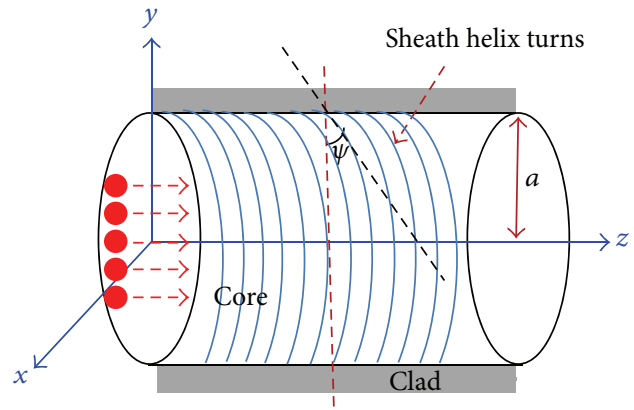

FIGURE 1: Longitudinal view of the guiding structure.

$$
\begin{aligned}
& E_{\varphi 2}=-\frac{j}{w^{2}}\left[\frac{j \beta v}{r} C K_{v}(w r)-\omega \mu w D K_{v}^{\prime}(w r)\right] e^{j(\omega t-\beta z+v \varphi)}, \\
& H_{\varphi 2}=-\frac{j}{w^{2}}\left[\omega \mu w C K_{v}^{\prime}(w r)+\frac{j \beta v}{r} D K_{v}(w r)\right] e^{j(\omega t-\beta z+v \varphi)},
\end{aligned}
$$

with $w=\sqrt{\beta^{2}-\left(n_{\text {clad }} k_{0}\right)^{2}}$. In the above set of equations, $\mu$ and $\varepsilon$ are, respectively, the permeability and the permittivity of the medium, $\omega$ is the angular frequency in the unbounded medium, $\beta$ is the propagation constant in the medium, $k_{0}$ is the free-space propagation constant, $v$ is the azimuthal mode index, and primes represent differentiation with respect to the arguments of field functions. Also $A, B, C$, and $D$ are unknown constants, to be estimated by the use of boundary conditions.

Now, considering the existence of DB twisted clad, boundary conditions will essentially include vanishing normal components of electric/magnetic fields and continuous tangential components of the same at the medium interface. Implementation of this ultimately leads to the evolution of a set of four homogeneous equations, followed by the dispersion relation (as obtained by collecting the coefficients of unknown constants) given as

$$
\begin{aligned}
f(\beta)= & \frac{1}{4} \omega^{2} \mu \varepsilon w^{2}\left\{K_{v-1}(a w)+K_{v+1}(a w)\right\}^{2} \sin ^{2} \psi \\
\times & {\left[\frac{v^{2} \omega^{2} \mu \varepsilon}{a^{2} u^{4}} J_{v}^{2}(a u)(\cos \psi-\sin \psi)^{2}\right.} \\
& \left.\quad-\frac{\beta^{2}}{4}\left\{J_{v-1}(a u)-J_{v+1}(a u)\right\}^{2}(\sin \psi-\cos \psi)^{2}\right] \\
+ & \left\{K_{v}(a w) \cos \psi+\frac{v \beta}{a w^{2}} J_{v}(a u) \sin \psi\right\} \\
\times & \left\{K_{v}(a w) \cos \psi-\frac{v \beta}{a w^{2}} K_{v}(a w) \cos \psi\right\} \\
\times & \left\{\frac{v^{2} \mu \varepsilon \omega^{2}}{a^{2} u^{4}} J_{v}^{2}(a u)-\frac{1}{4} \beta^{2}\left(J_{v-1}(a u)-J_{v+1}(a u)\right)^{2}\right\} \\
\times & \times(\sin \psi-\cos \psi)^{2}=0 .
\end{aligned}
$$


The unknown coefficients could be determined solving the aforesaid four equations obtained after implementing the boundary conditions. After some rigorous mathematical steps, the coefficients $A, B$, and $C$ can be determined in terms of the coefficient $D$ as follows:

$$
\begin{gathered}
A=-j \frac{u^{2} a J_{v}^{\prime}(u a) \Theta\left(u^{2} a \gamma \Delta-\Xi \xi\right)+w^{2} a\left(\Theta \xi-\omega \varepsilon K_{v}(w a)\right)}{\omega \varepsilon a w^{2} \gamma\left(\Theta \xi-\omega \varepsilon K_{v}(w a) \delta\right)} D, \\
B=\frac{u J_{v}(u a) \Theta\left(u^{2} a \gamma \Delta-\Xi \xi\right)+w^{2} a\left(\Theta \xi-\omega \varepsilon K_{v}(w a) \delta\right)}{w^{2} a \gamma\left(\Theta \xi-\omega \varepsilon K_{v}(a w) \delta\right)} D, \\
C=j \frac{u^{2} a \gamma \Delta-\omega^{2} \mu \varepsilon u w^{3} K_{v}^{\prime}(w a) \xi}{\omega \varepsilon K_{v}(a w)\left(w^{2} a \cos \psi-\beta v \sin \psi\right) \xi-\delta} D,
\end{gathered}
$$

where

$$
\begin{gathered}
\gamma=\omega^{2} \mu \varepsilon u^{2} J_{v}(u a) J_{v}^{\prime}(u a) \sin \psi \\
-\beta J_{v}^{\prime}(u a)\left(u^{2} a J_{v}(u a) \cos \psi+\beta v J_{v}(u a) \sin \psi\right), \\
\xi=J_{v}^{2}(u a)\left(u^{2} a \cos \psi+\beta v \sin \psi\right)-\beta u^{2} a^{2} J_{v}^{\prime}(u a) \sin \psi, \\
\Theta=\omega \epsilon K_{v}(w a)\left(w^{2} a \cos \psi-\beta v \sin \psi\right), \\
\Delta=w^{2} K_{v}(w a) \cos \psi+\beta v K_{v}(w a) \sin \psi, \\
\Xi=\omega^{2} \mu \varepsilon w^{3} K_{v}(w a) \sin \psi, \\
\delta=\omega \varepsilon u w a^{2} K_{v}(w a) \sin \psi .
\end{gathered}
$$

Now, the flux density in the guide along the direction of propagation can be estimated, and, following the procedure in [11], it will finally assume the forms corresponding to the core/clad sections, as follows:

$$
\begin{gathered}
\left(S_{z}\right)_{\text {Core }}=\frac{\beta}{2 u^{4}}\left(\varepsilon|A|^{2}+\mu|B|^{2}\right)\left\{\left(\frac{v}{r}\right)^{2} J_{v}^{2}(u r)+\left(\frac{u}{2}\right)^{2} \sigma\right\}, \\
\left(S_{z}\right)_{\text {Clad }}=\frac{\beta}{2 w^{4}}\left(\varepsilon|C|^{2}+\mu|D|^{2}\right)\left\{\left(\frac{v}{r}\right)^{2} K_{v}^{2}(w r)+\left(\frac{w}{2}\right)^{2} \Lambda\right\},
\end{gathered}
$$

with

$$
\begin{gathered}
\sigma=J_{v-1}^{2}(u r)+J_{v+1}^{2}(u r)-2 J_{v-1}(u r) J_{v+1}(u r), \\
\Lambda=K_{v-1}^{2}(w r)+K_{v+1}^{2}(w r)-2 K_{v-1}(w r) K_{v+1}(w r) .
\end{gathered}
$$

Equations (10) can be written in terms of only one unknown constant, for example, in terms of $D$, as determined by the use of (8).

\section{Results and Discussion}

We now investigate the propagation features of flux density through DB twisted clad microstructured guide. However, for this purpose, it remains essential to evaluate the allowed values of propagation constants corresponding to different sustained modes under the conditions when the DB twists are typically oriented perpendicular as well as parallel to
TABLE 1: Hybrid mode propagation constants.

\begin{tabular}{lllc}
\hline $\begin{array}{l}\text { Pitch angle } \\
\psi\end{array}$ & $\begin{array}{c}\text { Core } \\
\text { radius } a\end{array}$ & Mode & $\begin{array}{c}\text { Propagation constant } \\
\beta\left(\mathrm{m}^{-1}\right)\end{array}$ \\
\hline \multirow{3}{*}{$10 \mu \mathrm{m}$} & $\mathrm{EH}_{01}$ & $5.970 \times 10^{6}$ \\
& & $\mathrm{EH}_{11}$ & $5.975 \times 10^{6}$ \\
$0^{\circ}$ & $\mathrm{EH}_{21}$ & $5.968 \times 10^{6}$ \\
& & $\mathrm{EH}_{01}$ & $5.960 \times 10^{6}$ \\
& \multirow{2}{*}{$20 \mu \mathrm{m}$} & $\mathrm{EH}_{11}$ & $5.965 \times 10^{6}$ \\
& & $\mathrm{EH}_{21}$ & $5.970 \times 10^{6}$ \\
\hline \multirow{3}{*}{$10 \mu \mathrm{m}$} & $\mathrm{EH}_{01}$ & $5.850 \times 10^{6}$ \\
& & $\mathrm{EH}_{11}$ & $5.890 \times 10^{6}$ \\
& & $\mathrm{EH}_{21}$ & $5.870 \times 10^{6}$ \\
& & $\mathrm{EH}_{01}$ & $5.950 \times 10^{6}$ \\
& \multirow{2}{*}{$20 \mu \mathrm{m}$} & $\mathrm{EH}_{11}$ & $5.980 \times 10^{6}$ \\
& & $\mathrm{EH}_{21}$ & $5.965 \times 10^{6}$ \\
\hline
\end{tabular}

the direction of wave propagation. For the computational purpose, we consider the RI values of the core and the clad sections as 1.485 and 1.470 , respectively, and the operating wavelength is taken to be $1.55 \mu \mathrm{m}$. The chosen parameters are, indeed, the widely accepted ones in the case of modeling and simulations related to the electromagnetic properties of optical fibers. Also, we consider two typical values of core radius as $10 \mu \mathrm{m}$ and $20 \mu \mathrm{m}$, the clad radius being fixed at $100 \mu \mathrm{m}$. The behavior of normalized flux density through core/clad regions is studied considering three low-order hybrid $\mathrm{EH}_{01}, \mathrm{EH}_{11}$, and $\mathrm{EH}_{21}$ modes having propagation constants, as obtained by the use of eigenvalue (7), as shown in Table 1.

Figures 2(a) and 2(b), respectively, demonstrate the normalized flux density patterns through core and clad regions of guide having core radius $10 \mu \mathrm{m}$ under the situation when the DB twist is oriented perpendicular to the direction of propagation; that is, $\Psi=0^{\circ}$. In all the figures, the results corresponding to the $\mathrm{EH}_{01}, \mathrm{EH}_{11}$, and $\mathrm{EH}_{21}$ modes are represented by black dash-dot, solid red, and dashed blue lines. We observe from Figure 2(a) that the flux generally exhibits an oscillatory trend in the core region of the guiding medium. Also, in the central region of the core, $\mathrm{EH}_{11}$ hybrid mode has higher energy than the other two $\mathrm{EH}_{01}$ and $\mathrm{EH}_{21}$ modes. Further, flux densities due to the $\mathrm{EH}_{01}$ and $\mathrm{EH}_{11}$ modes decrease with the increase in radial distance, whereas that due to the $\mathrm{EH}_{21}$ mode exhibits a minor increase only.

In the clad section, as Figure 2(b) shows, the aforesaid three hybrid modes exhibit similar flux characteristics, and it remains extremely small as compared to that in the core region. At the core-clad interface, it shows relatively large value, and then step-falls with a little increase in clad radius, and become almost vanishing as the radius is further increased.

Figures 3(a) and 3(b) correspond to the situations when the DB helical windings are put parallel to the optical axis, that is, the helix pitch $\Psi=90^{\circ}$. We observe in this case that the confinements of flux in the core section (Figure 3(a)) exhibit almost similar patterns, as observed in the case when 


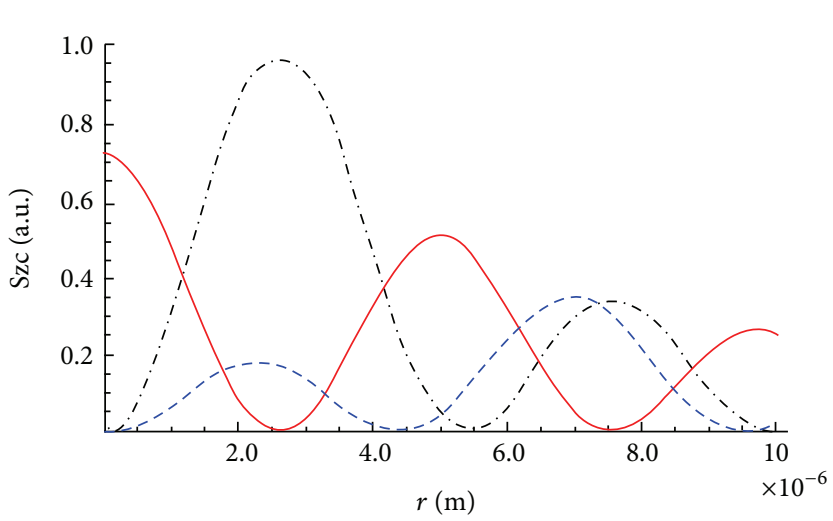

(a)

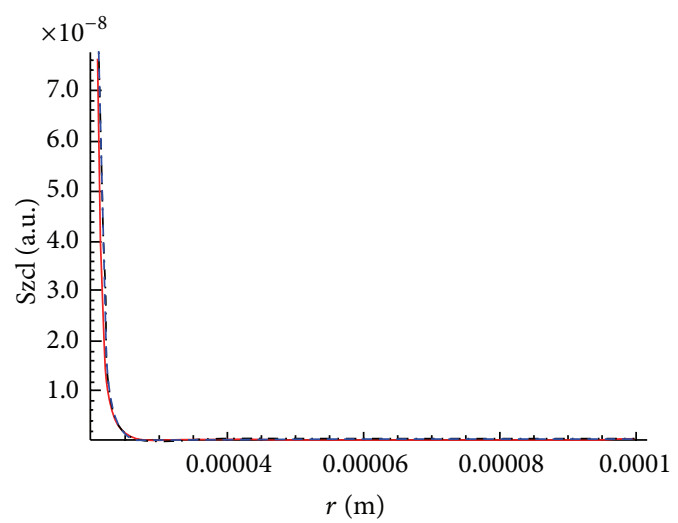

(b)

Figure 2: Flux density through the core (a) and clad (b) corresponding to $10 \mu \mathrm{m}$ core radius and $0^{\circ}$ helix pitch.

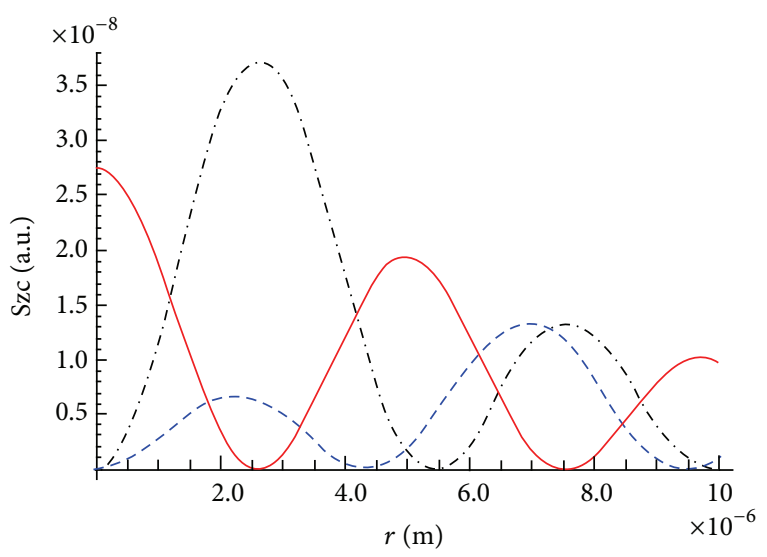

(a)

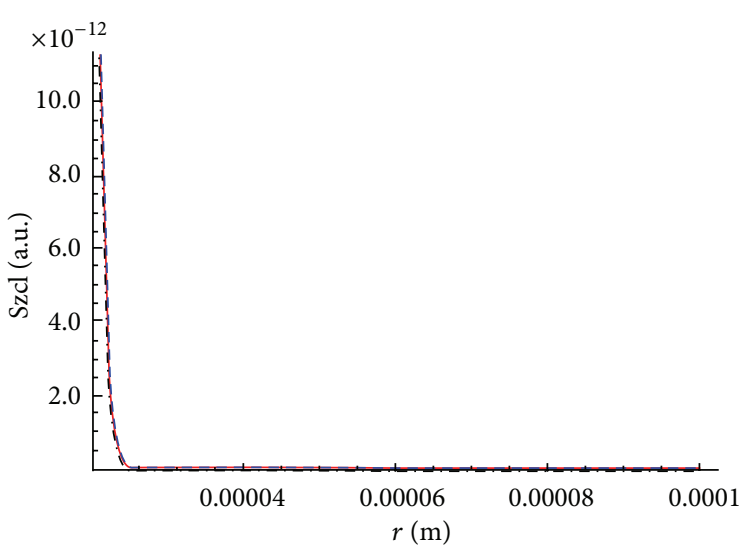

(b)

Figure 3: Flux density through the core (a) and clad (b) corresponding to $10 \mu \mathrm{m}$ core radius and $90^{\circ}$ helix pitch.

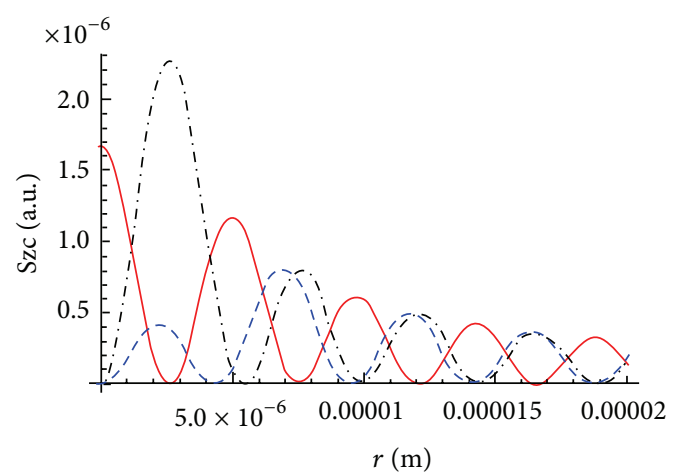

(a)

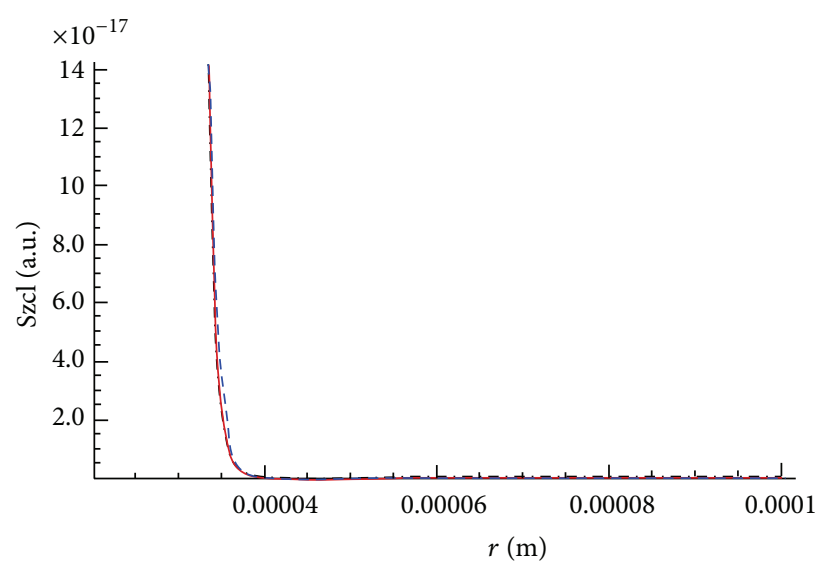

(b)

Figure 4: Flux density through the core (a) and clad (b) corresponding to $20 \mu \mathrm{m}$ core radius and $0^{\circ}$ helix pitch. 


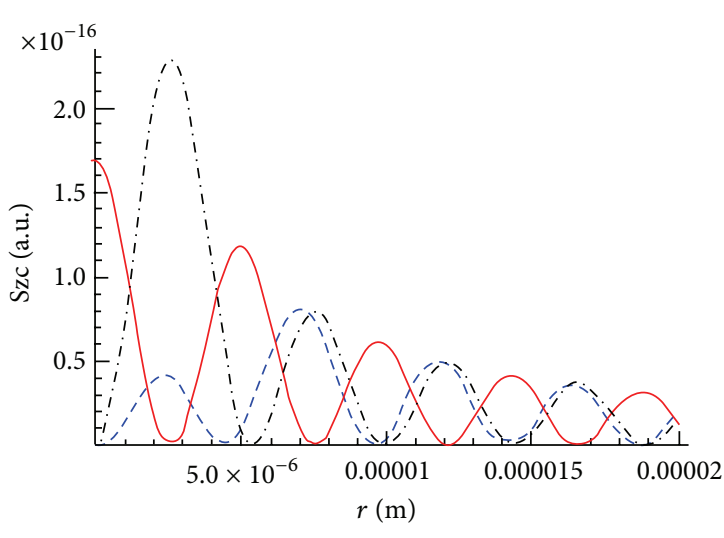

(a)

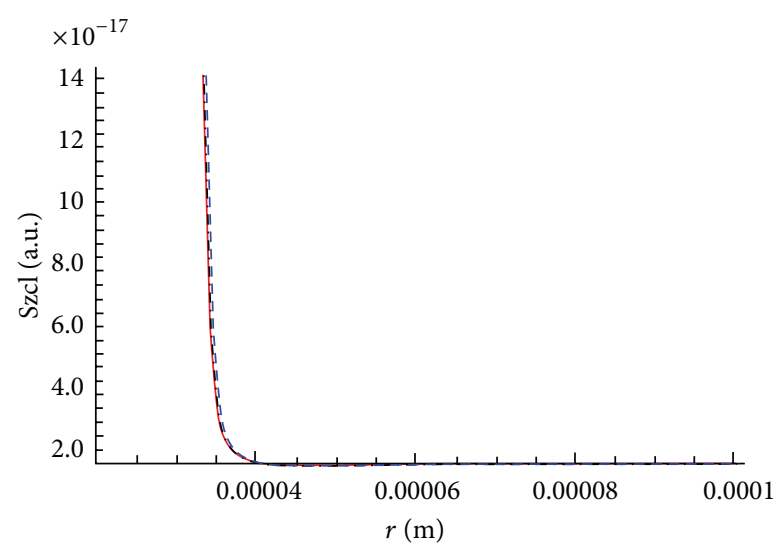

(b)

Figure 5: Flux density through the core (a) and clad (b) corresponding to $20 \mu \mathrm{m}$ core radius and $90^{\circ}$ helix pitch.

DB twists are oriented in the direction perpendicular to the wave propagation (Figure 2(a)), but its values are drastically reduced corresponding to all the sustained modes. Otherwise, the $\mathrm{EH}_{01}$ and $\mathrm{EH}_{11}$ modes correspond to higher power than the $\mathrm{EH}_{21}$ mode in the central region of core section. In the clad section too, the flux is further reduced (Figure 3(b)) as compared to the case of Figure 2(b), exhibiting similar density patterns, as observed before, for the situation of $\Psi=$ $0^{\circ}$. Thus, comparing the results of Figures 2 and 3, we notice the effect of introducing DB sheath helix structure at the dielectric core-clad interface. We find that making the angle of pitch (of DB helix) as $90^{\circ}$ greatly reduces the propagation of power, and therefore, a change in pitch would make the guide to amplify or attenuate the signal depending upon lowering or increasing its value. This clearly demonstrates the importance of such guiding structures.

Figures 4 and 5 correspond to the situation when the core radius is doubled, that is, increased to $20 \mu \mathrm{m}$. We observe that the trends of flux density patterns are almost similar to the situation as observed before for $10 \mu \mathrm{m}$, but the oscillations of flux are increased in this case. This is very much obvious due to more room for the fields to be sustained, thereby causing dispersion and so forth. However, in this as well, we observe that the increase of pitch of DB twist essentially reduces the flow of flux density, as evidenced by Figures 4 and 5 .

\section{Conclusion}

The eigenvalue equation is deduced for the aforesaid structure, and the propagation features of the low-order hybrid modes are estimated. Investigations are made of the energy flux density patterns corresponding to the sustained modes, and the effects are observed due to the alterations of pitch angles of the microstructured DB twists introduced at the dielectric core-clad interface. The flux patterns reveal profound effect of helix pitch, and making the DB windings parallel to the axis of guide greatly reduces power confinements in the guiding region. As such, these guides may be used for the purpose of amplifying and/or attenuating signals in optical systems.

\section{Conflict of Interests}

The authors declare that there is no conflict of interest regarding the publication of this manuscript.

\section{Acknowledgments}

The authors are grateful to Professors S. Shaari and B.Y. Majlis for constant encouragement and help. Also, they are thankful for the Ministry of Higher Education (Malaysia) for providing financial support to the work. The authors are grateful to the anonymous reviewer for making constructive criticisms.

\section{References}

[1] V. G. Veselago, "The electrodynamics of substances with simultaneously negative values of $\varepsilon$ and $\mu$," Soviet Physics Uspekhi, vol. 10, no. 4, pp. 509-514, 1968.

[2] J. B. Pendry, "Negative refraction makes a perfect lens," Physical Review Letters, vol. 85, no. 18, pp. 3966-3969, 2000.

[3] A. D. Yaghjian, "Extreme electromagnetic boundary conditions and their manifestation at the inner surfaces of spherical and cylindrical cloaks," Metamaterials, vol. 4, no. 2-3, pp. 70-76, 2010.

[4] N. Landy and D. R. Smith, "A full-parameter unidirectional metamaterial cloak for microwave," Nature Materials, vol. 12, pp. 25-28, 2013.

[5] A. J. Garcia-Collado, G. J. Molina-Cuberos, J. Margineda, M. J. Núnez, and E. Martín, "Isotropic and homogeneous behavior of chiral media based on periodical inclusions of cranks," IEEE Microwave and Wireless Components Letters, vol. 20, no. 3, pp. 175-177, 2010.

[6] G. J. Molina-Cuberos, Á. J. García-Collado, I. Barba, and J. Margineda, "Chiral metamaterials with negative refractive index composed by an eight-cranks molecule," IEEE Antennas and Wireless Propagation Letters, vol. 10, pp. 1488-1490, 2011.

[7] P. K. Choudhury and R. A. Lessard, "An estimation of power transmission through a doubly clad optical fiber with an annular core," Microwave and Optical Technology Letters, vol. 29, no. 6, pp. 402-405, 2001. 
[8] S. Tretyakov, I. Nefedov, A. Sihvola, S. Maslovski, and C. Simovski, "Waves and energy in chiral nihility," Journal of Electromagnetic Waves and Applications, vol. 17, no. 5, pp. 695-706, 2003.

[9] S. C. Yeow, M. H. Lim, and P. K. Choudhury, "A rigorous analysis of the distribution of power in plastic clad linear tapered fibers," Optik, vol. 117, no. 9, pp. 405-410, 2006.

[10] P. K. Choudhury and W. K. Soon, "On the transmission by liquid crystal tapered optical fibers," Optik, vol. 122, no. 12, pp. 10611068, 2011.

[11] M. A. Baqir and P. K. Choudhury, "On the energy flux through a uniaxial chiral metamaterial made circular waveguide under PMC boundary," Journal of Electromagnetic Waves and Applications, vol. 26, no. 16, pp. 2165-2175, 2012.

[12] U. N. Singh, O. N. Singh II, P. Khastgir, and K. K. Dey, "Dispersion characteristics of a helically cladded step-index optical fiber-analytical study," Journal of the Optical Society of America B, vol. 12, no. 7, pp. 1273-1278, 1995.

[13] D. Kumar and O. N. Singh II, "Modal characteristic equation and dispersion curves for an elliptical step-index fiber with a conducting helical winding on the core-cladding boundary-an analytical study," Journal of Lightwave Technology, vol. 20, no. 8, pp. 1416-1424, 2002.

[14] C. C. Siong and P. K. Choudhury, "Propagation characteristics of tapered core helical cald dielectric optical fibers," Journal of Electromagnetic Waves and Applications, vol. 23, no. 5, pp. 663674, 2009.

[15] K. Y. Lim, P. K. Choudhury, and Z. Yusoff, "Chirofibers with helical windings — an analytical investigation," Optik, vol. 121, no. 11, pp. 980-987, 2010.

[16] M. Ghasemi and P. K. Choudhury, "On the sustainment of optical power in twisted clad dielectric cylindrical fiber," Journal of Electromagnetic Waves and Applications, vol. 27, no. 11, pp. 13821391, 2013.

[17] I. V. Lindell and A. H. Sihvola, "Electromagnetic boundary and its realization with anisotropic metamaterial," Physical Review E, vol. 79, no. 2, Article ID 026604, 2009.

[18] I. V. Lindell, H. Wallén, and A. Sihvola, "General electromagnetic boundary conditions involving normal field components," IEEE Antennas and Wireless Propagation Letters, vol. 8, pp. 877880, 2009.

[19] I. V. Lindell and A. H. Sihvola, "Electromagnetic boundary conditions defined in terms of normal field components," IEEE Transactions on Antennas and Propagation, vol. 58, no. 4, pp. 1128-1135, 2010.

[20] I. V. Lindell, A. Sihvola, L. Bergamin, and A. Favaro, "Realization of the $\mathrm{D}^{\prime} \mathrm{B}^{\prime}$ boundary condition," IEEE Antennas and Wireless Propagation Letters, vol. 10, pp. 643-646, 2011.

[21] I. V. Lindell and A. H. Sihvola, "SHDB boundary condition realized by pseudochiral media," IEEE Antennas and Wireless Propagation Letters, vol. 12, pp. 591-594, 2013.

[22] M. A. Baqir and P. K. Choudhury, "Propagation through uniaxial anisotropic chiral waveguide under DB boundary conditions," Journal of Electromagnetic Waves and Applications, vol. 27, no. 6, pp. 783-793, 2013. 

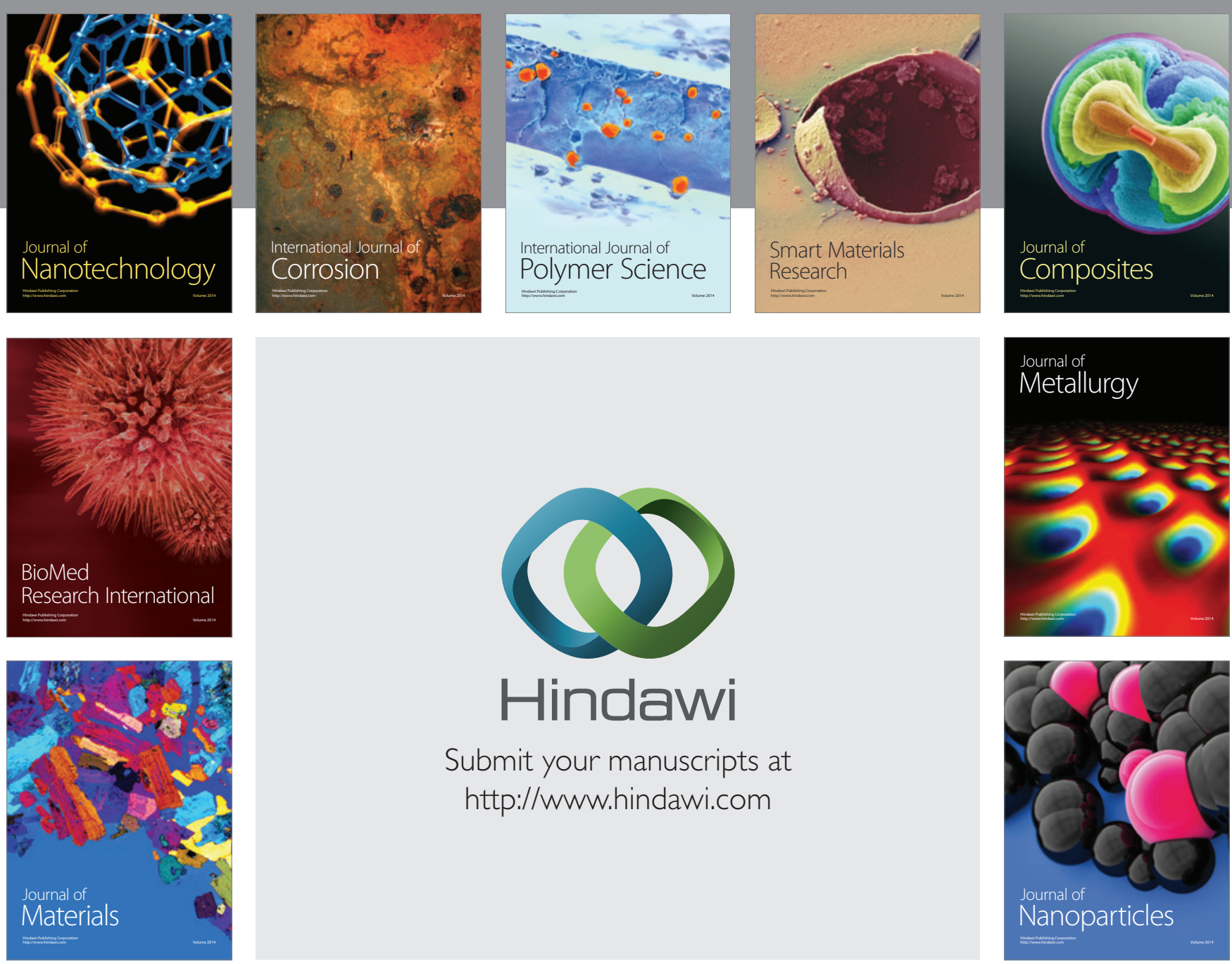

Submit your manuscripts at http://www.hindawi.com
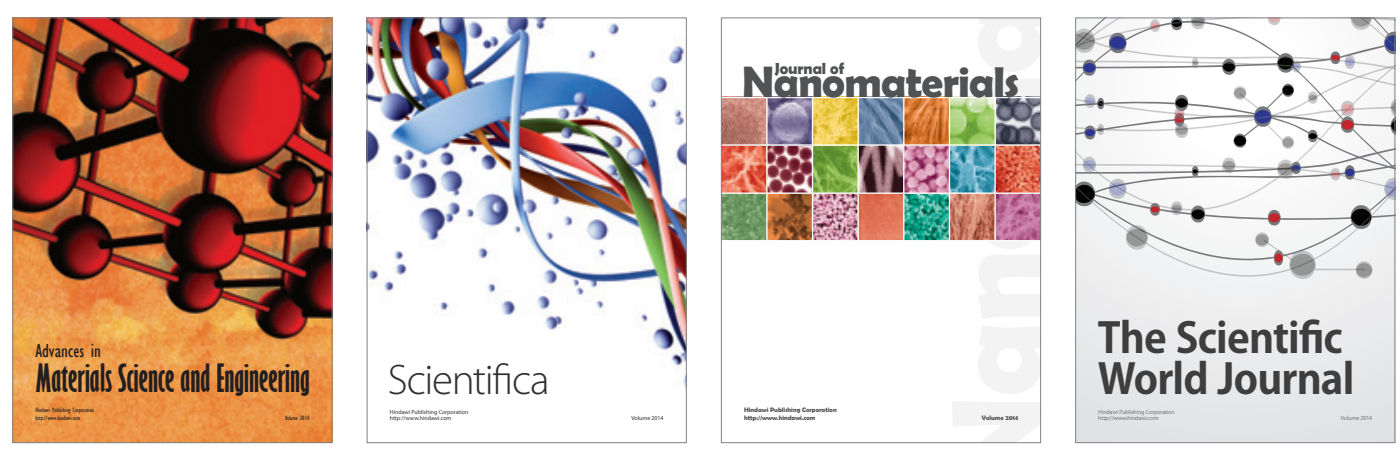

\section{The Scientific World Journal}
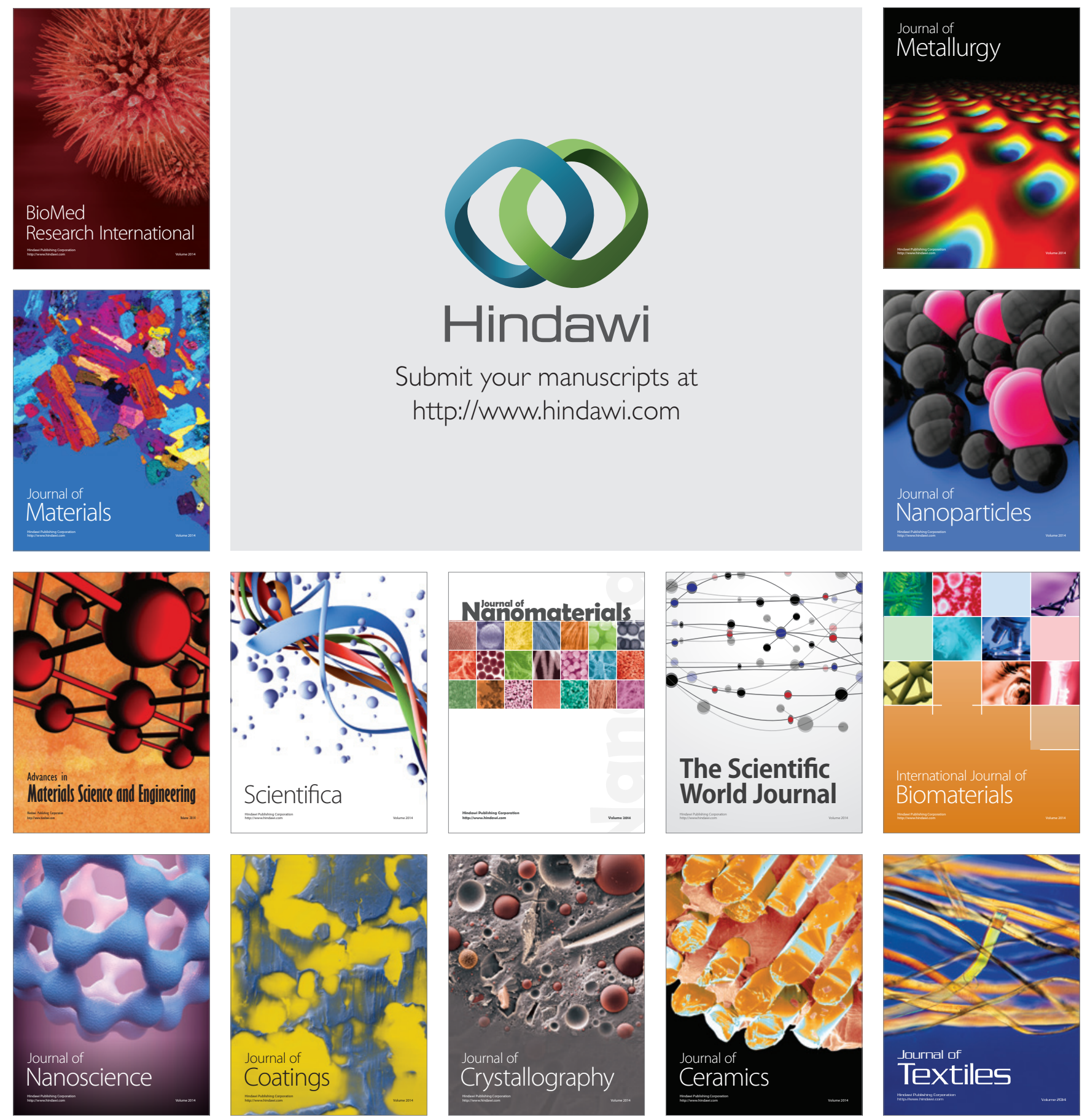